\title{
Damage Signature of Fatigued Fabric Reinforced Plastics in the Pulsed Ultrasonic Polar Scan
}

\author{
M. Kersemans • I. De Baere • J. Degrieck • K. Van Den \\ Abeele • L. Pyl • F. Zastavnik • H. Sol • W. Van Paepegem
}

Received: 20 March 2014 / Accepted: 22 July 2014 / Published online: 1 August 2014

(C) Society for Experimental Mechanics 2014

\begin{abstract}
This study investigates the use of both the amplitude and time-of-flight based pulsed ultrasonic polar scan (PUPS) for the nondestructive detection and evaluation of fatigue damage in fabric reinforced composites. Several thermoplastic carbon fabric reinforced PPS specimens (CETEX), loaded under various fatigue conditions, have been scanned at multiple material spots according to the P-UPS technique in order to extract material degradation in a quantitative way. The P-UPS results indicate that shear dominated fatigued carbon/PPS goes with a reduction of shear properties combined with large fiber distortions. The P-UPS results of the tension-tension fatigued carbon/PPS samples on the other hand reveal a directional degradation of the stiffness properties, reaching a maximum reduction of $-12.8 \%$ along the loading direction. The P-UPS extracted damage characteristics are fully supported by simulations, conventional destructive tests as well as visual inspection. The results demonstrate the excellent capability of the P-UPS method for nondestructively assessing and quantifying both shear-dominated and tension-tension fatigue damage in fabric reinforced plastics.
\end{abstract}

Keywords Pulsed ultrasonic polar scan · Fabric reinforced plastic $\cdot$ NDT $\cdot$ Fatigue damage characterization

M. Kersemans $(\triangle) \cdot$ I. De Baere $\cdot$ J. Degrieck $\cdot$ W. Van Paepegem Department of Materials Science and Engineering, Ghent University, Technologiepark-Zwijnaarde 903, 9052 Zwijnaarde, Belgium e-mail: mathias.kersemans@ugent.be

K. Van Den Abeele

Department of Physics, Catholic University of Leuven - KULAK, Etienne-Sabbelaan 52, 8500 Kortrijk, Belgium

L. Pyl $\cdot$ F. Zastavnik $\cdot$ H. Sol

Department Mechanics of Materials and Constructions, Vrije

Universiteit Brussel, Pleinlaan 2, 1050 Brussels, Belgium

\section{Introduction}

The excellent high-strength-to-low-mass ratio of fiber reinforced plastics, combined with their tunable elasticity and easy shaping of complex structures, makes them very interesting for use in high-tech applications demanding particular requirements, such as aerospace, wind turbines as well as others. Most often the mechanical nature of a composite material is anisotropic, demanding sophisticated inspection tools to affirm its design features, as well as to ascertain its mechanical and structural integrity in their life-time. The latter is of utmost importance considering that in-service components are subjected to a variety of loading conditions such as tensile, shear, fatigue and impact, effectively degrading the mechanical performance of the component. Several non-destructive testing techniques are already present in literature [1-14], of which ultrasonics can be considered to be the largest class. Though, none of them is fully capable to detect, discern and follow up different types of damage. Ultrasonic evaluation of geometrical defects, such as delaminations, macro cracks, etcetera, in fiber reinforced plastics is already well established [3, 15-20]. Basically the damaged area acts as an interface for the ultrasonic wave which leads to a scattering phenomenon, exposing the damaged area. Though, it is well known for fiber reinforced plastics that loading, and in particular fatigue loading, has an elasticity related damage signature. Indeed, fatigue loading leads to the initiation, progression and accumulation of micro defects, which manifest themselves in a directional reduction of the stiffness properties at the macro level [21-27]. Hence, to fully assess fatigue damage in fiber reinforced plastics, a sophisticated non-destructive inspection technique, capable of quantifying a directional elasticity reduction, is required.

The pulsed ultrasonic polar scan (P-UPS) technique [28] has already been demonstrated to be a promising means for NDT and material characterization. Basically, the method 
interrogates a material spot with pulsed ultrasound from every possible angle of incidence $\psi(\varphi, \theta)$, with $\varphi$ the azimuthal angle, further called the polar angle, and $\theta$ the angle with the normal on the surface, further called the incident angle (see Fig. 1(a)). To enhance coupling of acoustical energy into the solid under investigation, water is used as immersion liquid. Simply mapping the transmitted (or if necessary the reflected) amplitude or its associated time of flight (TOF) of the ultrasonic pulse as a function of the incidence angle $\psi(\varphi, \theta)$ in a polar representation, yields a P-UPS image in which intriguing patterns can be observed (see Fig. 1(b)). These characteristic contours put on view bulk wave characteristics and, consequently provide a fingerprint of the elasticity of the investigated material $[29,30]$. Early results obtained with the P-UPS technique include the detection of fiber direction $[28,29,31]$, determination of the fiber volume fraction [29, $31]$ and the detection of different porosity levels [29, 31]. More recently, the P-UPS has been successfully employed to characterize the elasticity tensor of orthotropic carbon fiber reinforced plastics by means of a genetic inversion procedure [32, 33]. In addition, (fiber reinforced) materials with degraded mechanical properties have also been investigated with the P-UPS technique [34-37]. As such, P-UPS was proven a useful means for (i) detection of delaminations [34], (ii) the assessment of degradation induced by shear dominated quasistatic hysteresis loading (a small number of sequential loading-unloading cycles) on various fiber reinforced plastics [35], (iii) the semi-quantification of early stage (hidden) corrosion [36], (iv) the detection and even localization of a (hidden) closed surface crack in a laminated glass panel [36] and (v) characterization of multidirectional (sub) surface corrugations [37]. Hence, it is clear that the P-UPS technique is a useful means for the inspection of various materials in different mechanical health. However, the application of the P-UPS technique for nondestructive detection and evaluation of fatigue damage in fiber-reinforced plastics has hardly been investigated. Most fiber-reinforced composites show a progressive directional reduction in stiffness properties upon fatigue loading through fiber/matrix interface debonding, matrix cracks, fiber bridging/pull-out/failure on the micro-scale $[21,22,25,27]$. There where most of the existing NDT tools fail to assess the effect of fatigue damage in composites, the nature and design of the P-UPS method should perfectly match such kind of material degradation.

Here, we investigate the feasibility of the P-UPS method (amplitude and TOF) to nondestructively assess both shear-dominated and tension-tension fatigue. The material under investigation is a carbon fabric reinforced plastic, which is already used as a structural component in the Airbus A320 and A380. It is clear that those components are subject to fatigue loading during their lifetime, emphasizing the necessity to understand, and even monitor, their material response.

\section{Materials and Procedure}

Several carbon fabric reinforced polyphenylene sulphide (PPS), called CETEX (Ten Cate, The Netherlands), have been subjected to dynamic fatigue loading, both in tensile and shear. The fiber type is the carbon T300J $3 \mathrm{~K}$ and the weaving pattern is a 5 -harness satin weave with a mass per surface unit of $286 \mathrm{~g} / \mathrm{m}^{2}$. Tension-tension loading has been applied on two stacking sequences, $\left[+45^{\circ},-45^{\circ}\right]_{4 \mathrm{~s}}$ and $\left[\left(0^{\circ}, 90^{\circ}\right)\right]_{4 \mathrm{~s}}$, each having a total thickness of $2.5 \mathrm{~mm}$. The fatigue tests have been performed on a servohydraulic Instron 8800 .

The $\left[+45^{\circ},-45^{\circ}\right]_{4 \mathrm{~s}}$ sample is cut at a length of $250 \mathrm{~mm}$ and a width of $30 \mathrm{~mm}$, and has been subjected to tension-tension loading at a frequency of $2 \mathrm{~Hz}$, which thus corresponds to shear dominated fatigue. The fatigue loading, with shear stress range $[0,50 \mathrm{MPa}]$, has been manually stopped after $\pm 135,000$ cycles without fracture of the sample.

Several $\left[0^{\circ}, 90^{\circ}\right]_{4 \mathrm{~s}}$ carbon/PPS samples with a dumbbell shape have been prepared. Recently, it has been shown that this specific geometry shows an excellent performance during tension-tension fatigue for this specific material [38]. The specimens, with ultimate tensile strength UTS $\approx 770 \mathrm{MPa}$, have been subjected to different tension-tension fatigue cycles at various load levels, until sudden failure of the specimens occurred:

Sample 155 load cycles with stress range [0-700 MPa] at a frequency of $2 \mathrm{~Hz}$

Sample 2 99,123 load cycles with stress range [0-650 MPa] at a frequency of $5 \mathrm{~Hz}$

Sample 3 314,111 load cycles with stress range [0-625 MPa] at a frequency of $5 \mathrm{~Hz}$

Where possible, the specimens have been equipped with a clip-on extensometer and thermocouple. As such, the axial strain, load, actuator displacement and temperature have been measured simultaneously.

After applying the fatigue cycles, the samples have been demounted from the tensile machine and placed in the P-UPS setup to obtain experiments in transmission. The scanner is equipped with two broadband unfocused piezoelectric transducers (General Electric), producing short bursts of ultrasound at a central frequency of $f_{c}=2 \mathrm{MHz}$. This frequency has been selected based on the damping characteristics of the carbon/ PPS material. A higher central frequency is not advisable because this would lead to a largely reduced transmission characteristic, making the extraction of material features, and in extension the assessment of fatigue damage, more difficult. The angular range for the incident angle $\theta$ is at least $\left[-65^{\circ},+\right.$ $65^{\circ}$ ], with a fixed resolution of $0.05^{\circ}$, while the range for the polar angle $\varphi$ is set to $\left[0^{\circ}, 180^{\circ}\right]$ having an angular resolution of $0.5^{\circ}$. Hence, a single P-UPS experiment consists of around one million unique directions of insonification. 


\section{Physical Background P-UPS}

In this section, a short physical background of the P-UPS is provided, together with numerical simulation results in order to ease the interpretation of further experiments, as well as to have a means of validation.

In general, the inner contour of a P-UPS image is related to the quasi-longitudinal (QL) lateral wave (see Fig. 1(b)), and as such it is dominated by the in-plane stiffness properties of the material under investigation. The outer contours relate to the quasi-shear horizontal (QSH) and quasi-shear vertical (QSV) lateral waves and are mainly dictated by shear properties [39]. For example, the elliptical nature of the inner contour in Fig. 1(b) is induced by the unidirectional fiber reinforcement of the $[0]_{8}$ unidirectional carbon/epoxy laminate. Indeed, the reinforcement fibers result in a directional in-plane stiffness $C_{i i}$, reaching a maximum, respectively minimum value along $\varphi=0^{\circ}$, respectively $\varphi=90^{\circ}$. As such, the phase velocity of the in-plane propagating QL-wave becomes function of the polar angle $\varphi[35,40]$. By taking into account Snell-Descartes' law for externally born sound, which states that the components of the incident wave vector $k$, parallel to the liquid-solid interface (12-plane), are conserved, the incident angle $\theta$ for stimulating the in-plane propagating QL wave is dependent on the orientation of the insonification plane. Basically, a higher stiffness $C_{i i}$ results in a lower critical (incident) angle $\theta$, and vice versa $[35,40]$. With this, the geometrical appearance of the QL contour in Fig. 1(b) can be easily interpreted: the inward part of the contour (along $\varphi=0^{\circ}$ ) corresponds to a high elastic stiffness, precisely because of the orientation of the inserted fiber reinforcement, while the wider part of the contour corresponds to a lower stiffness value, mainly reflecting the low elasticity of the matrix of the composite. It is clear that the same applies for the two outer, shear dominated P-UPS contours. Hence, any change in the elasticity tensor alters the directional wave speed and thus yields a positional shift of the P-UPS contours. In order to visually demonstrate this, P-UPS simulations [30, 41, 42] are performed for a virgin and degraded carbon/PPS fabric when considering pulsed ultrasound (see Fig. 2). Here, we model the fabric as if it consists of 16 unidirectional laminae stacked in a $\left[0^{\circ}, 90^{\circ}\right]$ sequence. The complex-valued symmetrical stiffness tensor $C=C^{\prime}+i C^{\prime \prime}$ for each lamina is listed in Table 1. The real part corresponds to the mechanical stiffness, while the imaginary part corresponds to a viscous damping term to account for acoustic losses (Kelvin-Voigt solid) [40]. It has been verified that the P-UPS simulation for a carbon/PPS fabric with homogenized elasticity constants (see table 4 in reference [43]) basically shows equivalent features as our current modeling approach. This can be easily understood because the large number of thin laminae has a homogenization effect for ultrasonic waves. For simulating fiber distortions, the current modeling approach is found to be advantageous.

The simulation for the virgin carbon/PPS material is shown in Fig. 2(a). Note that the contour associated with the shear vertically polarized bulk wave could not be captured within the operational range of the scanning apparatus because of low $G_{13}^{\prime}$ and $G_{23}^{\prime}$ values. Nevertheless, since the immersion liquid acts as a 'lens' for the P-UPS [39], one could employ a different liquid in order to get a zoomed out P-UPS image. However, this is not considered in the present study. The effect of a $30 \%$ reduction of stiffness $E_{11}^{\prime}$ in the $0^{\circ}$-laminae (parameter $p=0.7$ and $q=1$ in Table 1) is shown in Fig. 2(b). Reduction of the shear modulus $G_{12}^{\prime}$ by $30 \%$ (parameter $p=1$ and $q=0.7$ in Table 1) is displayed in Fig. 2(c). Figure 2(d) represents the PUPS image when, in addition to the reduction of $G_{12}^{\prime}$, a fiber distortion of $8^{\circ}$ is considered. It is clear that these features are well represented in the P-UPS image and can be easily identified by the positional shifting of the P-UPS contours.

\section{Results and Discussion}

\section{Shear-Dominated Fatigue Loading}

The experimental P-UPS results for the virgin $\left[+45^{\circ},-45^{\circ}\right]_{4 \mathrm{~s}}$ carbon/PPS sample are presented in Fig. 3. The geometrical
Fig. 1 Schematic representation of the P-UPS principle (a) and amplitude recorded P-UPS for a $\left[0^{\circ}\right]_{8}$ unidirectional carbon/epoxy laminate (b). The added indicators identify the characteristic PUPS contours which reflect the elastic material properties
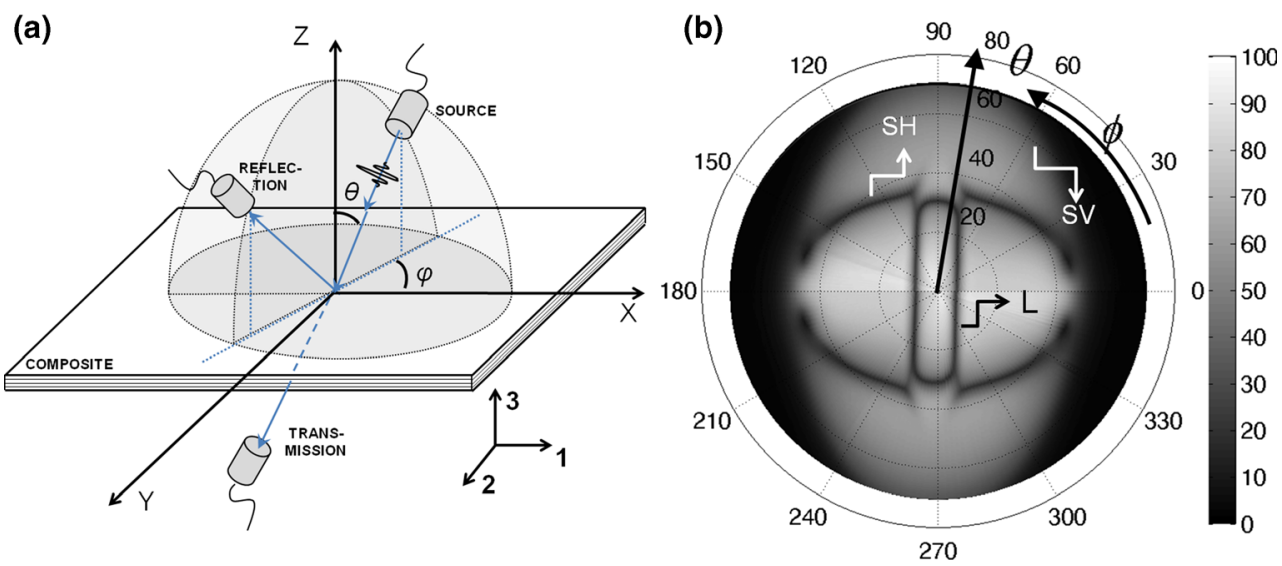
Fig. 2 P-UPS simulation of carbon/PPS fabric (material parameters are listed in Table 1) with thickness $2.5 \mathrm{~mm}$ at frequency $f_{c}=2 \mathrm{MHz}:\left[0^{\circ}, 90^{\circ}\right]_{4 \mathrm{~s}}$ with $p=$ $q=1$ (virgin material) (a), $\left[0^{\circ}, 90^{\circ}\right]_{4 \mathrm{~s}}$ with $p=0.7$ and $q=1$ (b), $\left[0^{\circ}, 90^{\circ}\right]_{4 \mathrm{~s}}$ with $p=1$ and $q=$ 0.7 (c) and $\left[8^{\circ}, 82^{\circ}\right]_{4 \mathrm{~s}}$ with $p=1$ and $q=0.7$ (d)
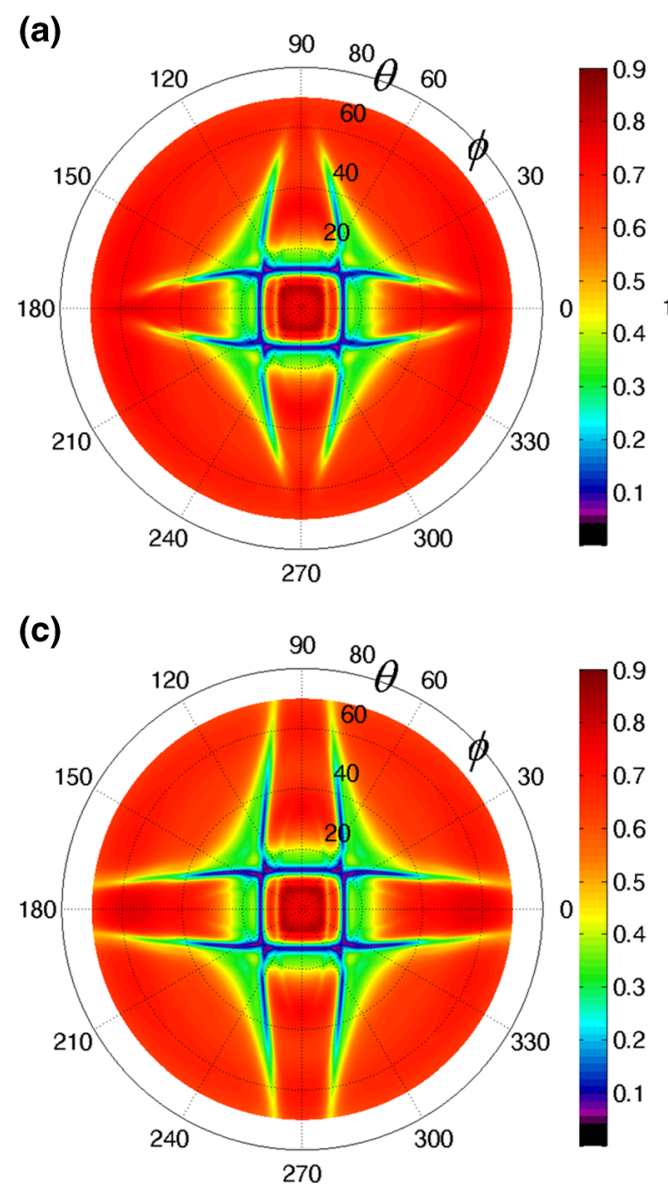

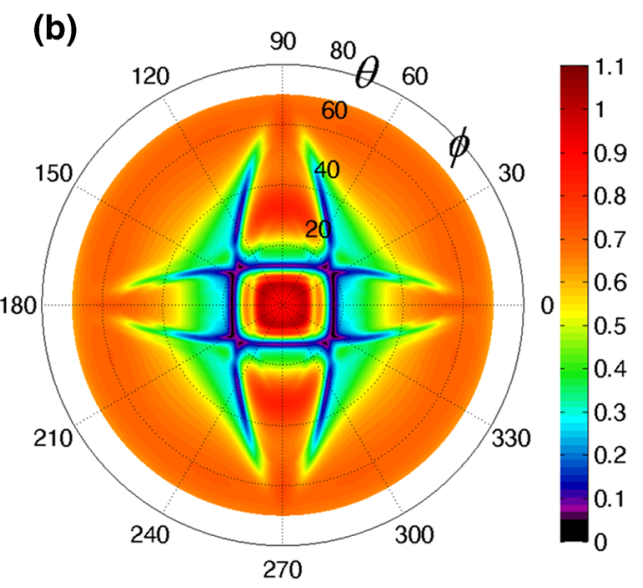

(d)

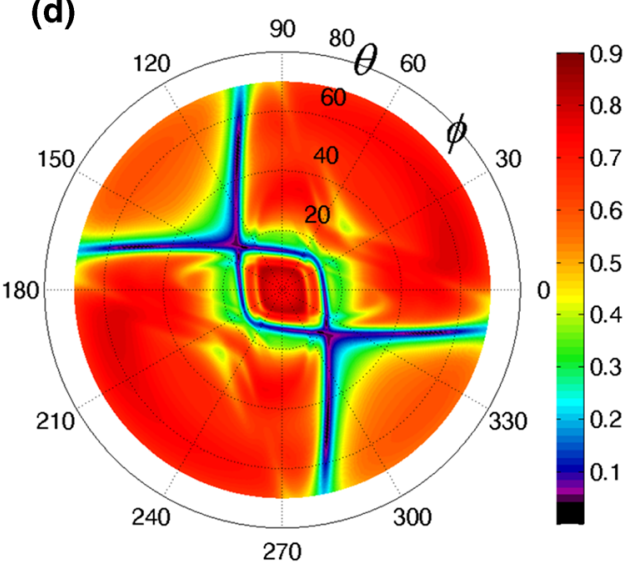

shape of the P-UPS image reflects the perfect orthotropic nature of the investigated carbon/PPS sample, i.e. the principal axes of orthotropy coincide with the fiber reinforcement structure.

The $\left[+45^{\circ},-45^{\circ}\right]_{4 \mathrm{~s}}$ fabric has first been subjected to running-in load cycles: the desired shear stress level of $50 \mathrm{MPa}$ is achieved by cyclic loading and unloading at the frequency $f=2 \mathrm{~Hz}$ while gradually increasing the tensile force (see Fig. 4(a)). After this initiation process, the fatigue loading itself takes place until the test has been manually stopped after $\pm 135,000$ load cycles without failure of the specimen. The variation in longitudinal strain $\varepsilon_{x x}$, as well as the temperature evolution during fatigue is shown in Fig. 4(b). The sudden temperature rise near the end of the fatigue loading originates from the internal friction of both multiple matrix cracks and the yarns at cross-over points. Together with the temperature $T$, the longitudinal strain evolves in a similar way, which is a strong indicator that the material effectively underwent severe damage. A photograph of the specimen after fatigue loading is shown in Fig. 4(c), here necking of the specimen can be clearly observed. Both TOF and amplitude P-UPS have been obtained at four different spots, which basically can be categorized from little visual damage (A and B) over reasonable visual damage $(C)$ to severe visual damage $(D)$.
With respect to the P-UPS results of the virgin material, the results at scan location A (see Fig. 5(a-b)) do not reveal any significant changes, implying that the material can be considered to be locally intact, in accordance with visual inspection.

This is clearly not valid anymore for scan location B (Fig. 5(c-d)). The inner QL-contour does not resemble a square anymore, but is rhomboid. In addition, the amplitude recorded P-UPS reveals that the cross-like contour, which is

Table 1 Material properties, with $p$ and $q$ parameters, used for the P-UPS simulations shown in Fig. 2

\begin{tabular}{|c|c|c|c|}
\hline Liquid & & Solid & \\
\hline \multirow[t]{8}{*}{$K[G P a]$} & 2.2 & $E_{11}[G P a]$ & $103 \cdot(p-0.06 i)$ \\
\hline & & $E_{22}[G P a]$ & $13.7 \cdot(1-0.4 i)$ \\
\hline & & $E_{33}[\mathrm{GPa}]$ & $13.7 \cdot(1-0.04 i)$ \\
\hline & & $\nu_{12}=\nu_{13}[-]$ & $0.29 \cdot(1-0.02 i)$ \\
\hline & & $\nu_{23}[-]$ & $0.35 \cdot(1-0.04 i)$ \\
\hline & & $G_{12}[G P a]$ & $4.28 \cdot(q-0.05 i)$ \\
\hline & & $G_{13}[G P a]$ & $3.1 \cdot(1-0.02 i)$ \\
\hline & & $G_{23}[G P a]$ & $3.1 \cdot(1-0.02 i)$ \\
\hline$\rho\left[\mathrm{kg} / \mathrm{m}^{3}\right]$ & 1000 & $\rho\left[\mathrm{kg} / \mathrm{m}^{3}\right]$ & 1600 \\
\hline
\end{tabular}


Fig. 3 P-UPS result for virgin [+ $\left.45^{\circ},-45^{\circ}\right]_{4 \mathrm{~s}}$ carbon/PPS fabric: amplitude (a) and TOF (b)

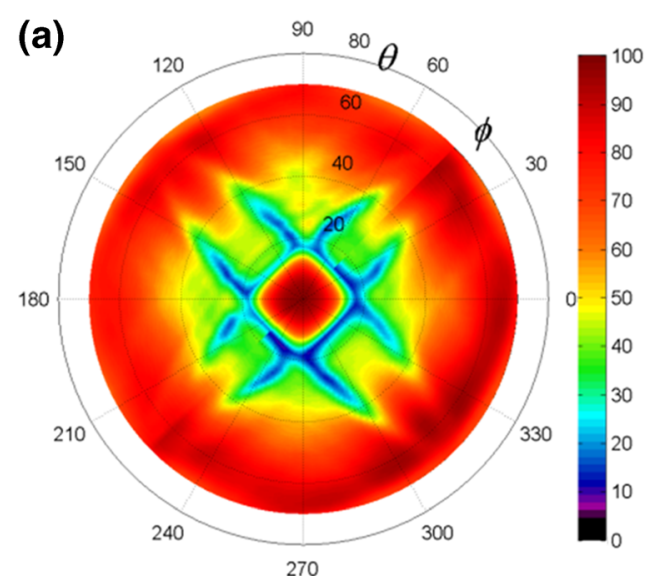

connected to the shear properties, is subjected to both a shortening and lengthening of its branches. This indicates that the initially perfect orthotropic material locally transformed to a lower symmetry class.

These indicators become even more outspoken at scan location $\mathrm{C}$, both in the amplitude and the TOF recording

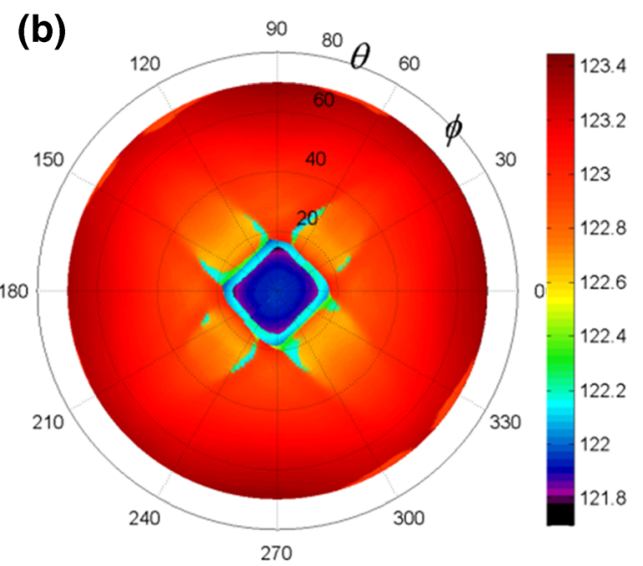

Fig. 4 Running-in load cycles (a), evolution of longitudinal strain $\varepsilon_{x x}$ and temperature $T$ during fatigue loading (b) and photograph of shear dominated fatigued carbon/PPS fabric (c). The labels denote the P-UPS scan locations, while the added coordinate systems coincides with the polar direction in the corresponding $\mathrm{P}$ UPS image (a)

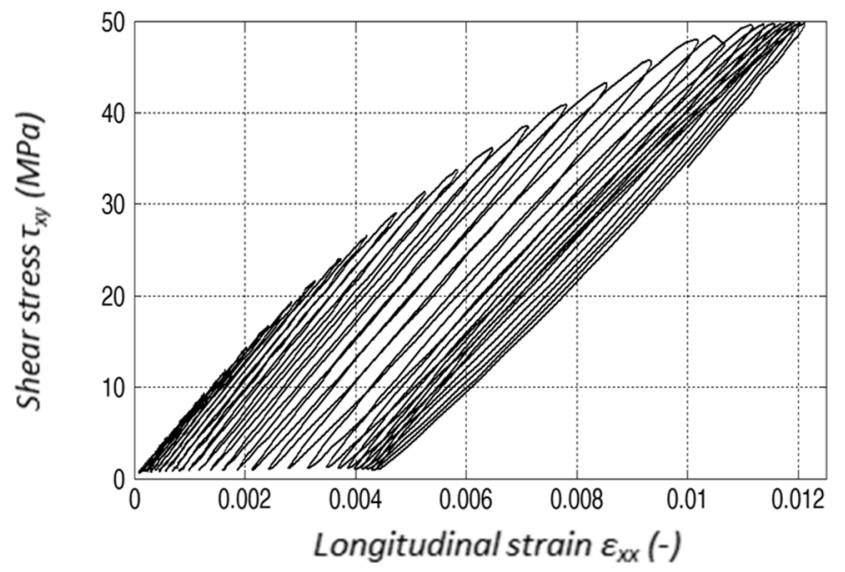

(b)

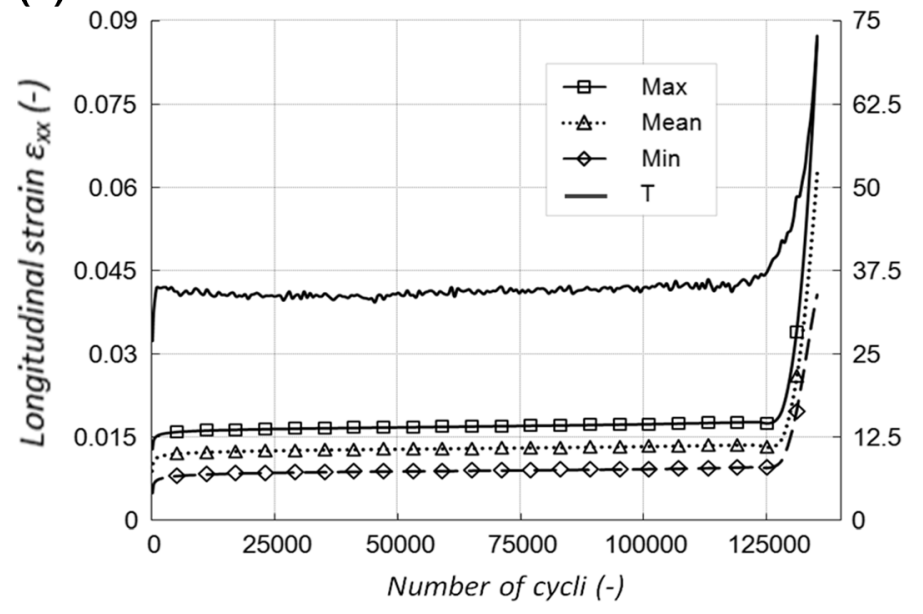

(c)

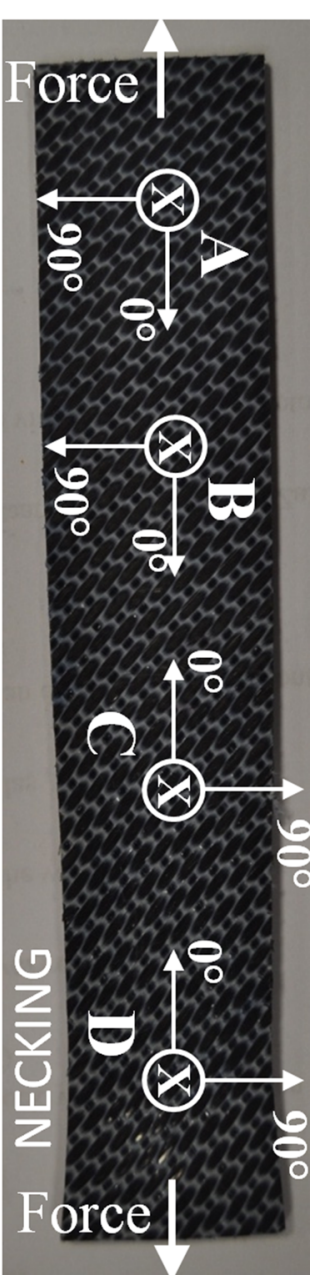


Fig. 5 P-UPS results for a shear dominated fatigued $\left[+45^{\circ},-45^{\circ}\right]_{4 \mathrm{~s}}$ carbon/PPS fabric: amplitude recording (left column) and TOF recording (right column). Material spot A (a-b), material spot B (cd), material spot $\mathrm{C}(\mathbf{e}-\mathbf{f})$ and material spot D (g-h) (a)

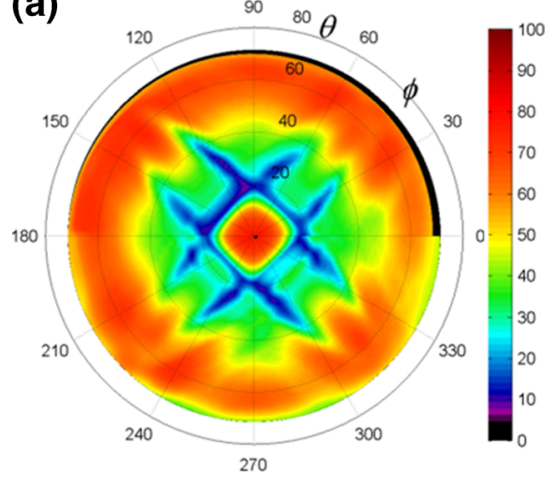

(c)

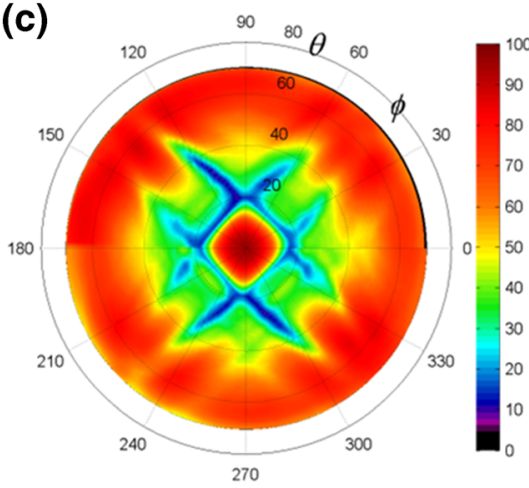

(e)

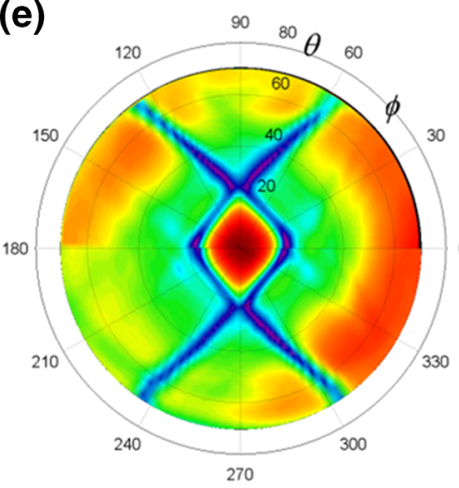

(g)

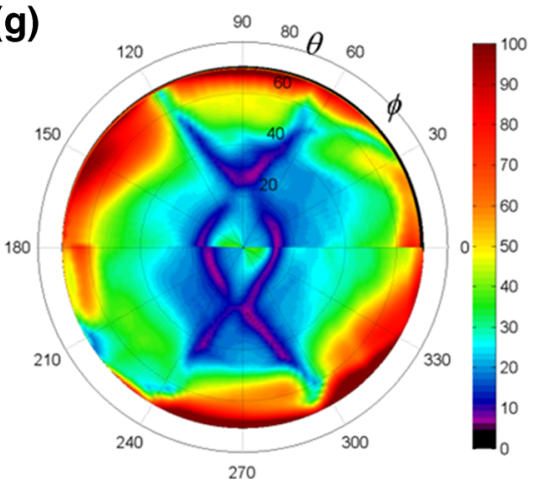

(b)
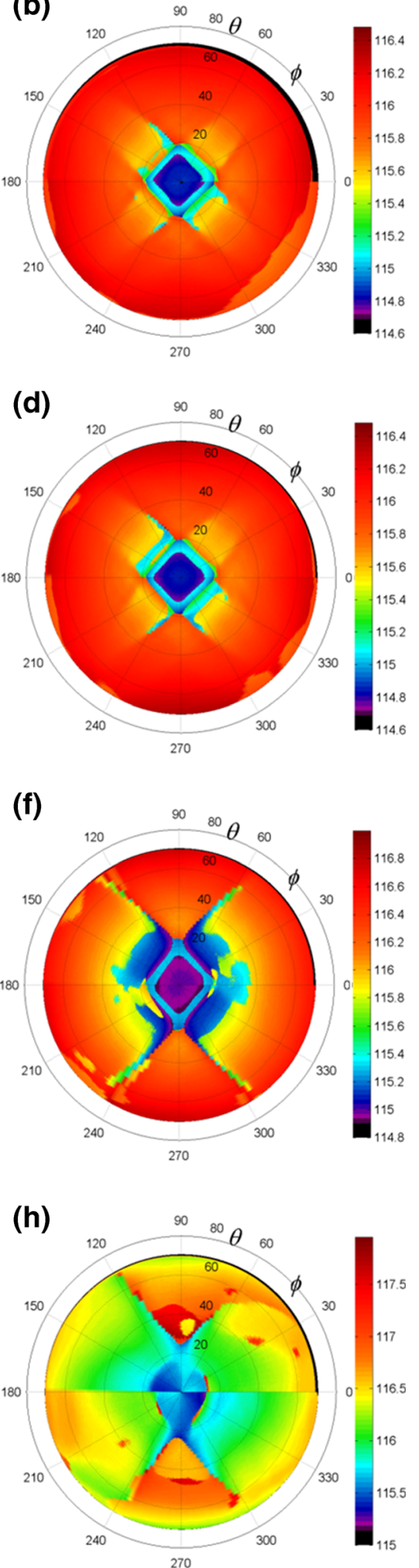

section II is still valid for damaged materials, this implies that the global stiffness of the sample in that particular direction increases. On the contrary, along $\varphi=90^{\circ}$ the opposite takes place as the inner contour widens, i.e. the loading induces a directional weakening of the sample. This directional strengthening and weakening of the material is easily understood when considering the large distortion of the fibers upon shear loading. Secondly, the observed phenomena in the 
shear-related contour become very prominent. Although some minor artifacts are left from the 8 branches of the original cross-like contour, the contour basically transformed its topology into 4 symmetric long branches, further indicating the directional nature of the damage. Note the good agreement with the numerical result (rotated over $45^{\circ}$ ) displayed in Fig. 2(d). It is also interesting to note that incidence angles which were initially equivalent $(\psi(\varphi, \theta)$ and $\psi(\varphi+\pi, \theta))$ in the amplitude map, are not equivalent anymore. Indeed, the recorded amplitude map does not possess any point symmetry anymore. This can be understood by considering that the scanned material spot has a slightly corrugated surface due to damage accumulation, which makes the coupling efficiency of the ultrasonic waves dependent upon the exact entrance angle. It is further noted that the time scale of the TOF recorded P-UPS spans a larger range. In other words, in some material directions, the ultrasonic signal needs more time to traverse the sample, and thus propagates at a lower rate. Obviously, this is an additional indicator of the presence of material damage.

Finally, the most heavily damaged spot (D) is investigated. The distortion of the local fiber orientation becomes even worse, and reaches a maximum rotation of more or less $13^{\circ}$. With X-ray, fiber distortions in the same order of magnitude have already been measured for composites loaded in a quasistatic shear state [44]. Note that the extracted distortion is an average since each layer has its own distortion level. In fact, the broadening (in $\theta$-direction) of the inner contour in the amplitude recorded P-UPS suggests that this is exactly what is happening. The appearance of such large fiber distortions can be understood by realizing that the carbon/PPS material belongs to the class of thermoplastics. Hence, the temperature rise during cyclic loading (see Fig. 4(b)) brings the PPS matrix close to its softening temperature $\left(\mathrm{T}_{\mathrm{g}} \approx 85{ }^{\circ} \mathrm{C}\right)$, making it relatively easy for the fibers to realign upon loading. For similar composites, we have measured temperatures up to $110{ }^{\circ} \mathrm{C}$ at the necking area. The extracted values for the distorted local fiber distribution are listed in Table 2 for all scan positions. Since the surface of the sample is marked with a characteristic pattern of the weaving pattern (see Fig. 4(c)), we also determined optically the local fiber distortion of the outer layers. However, note that the optically determined values are only valid for the outer layers, and thus could deviate from the actual internal fiber distribution.
The amplitude recorded P-UPS further shows that the transmission field, which is mainly dominated by QL polarized waves, drops in amplitude level for small incident angles $\left(\theta<20^{\circ}\right)$, thus making the sample QL opaque. This is most probably related to scattering of QL polarized waves on damage related geometrical discontinuities [35]. However, the opposite is observed for large incident angles $\left(\theta>60^{\circ}\right)$. The transmission field, which is now dominated by QSV polarized waves, reveals a significant increase in transmitted amplitude. In fact, saturation is observed. Hence, it seems that the induced fatigue damage enhances the transmission properties of QSV polarized waves, making the sample close to QSV transparent. This is quite odd, and might be due to the high temperature level attained during loading which weakens the PPS matrix and as a consequence might influence its chemical properties. Unfortunately, this statement could not be verified. The TOF recorded P-UPS at spot D reveals a further increase of the range of the time scale. For the virgin material, the range amounts $\Delta t=1.85 \mu \mathrm{s}$, while material spot $\mathrm{D}$ shows a range of $\Delta t=2.85 \mu \mathrm{s}$. Note that the increased travel time mainly manifests itself in certain material orientations, which again accentuates the directional nature of the induced fatigue damage.

The present results clearly show that the induced damage has a very local characteristic, i.e. the damage level gradually increases towards the necking zone, while it possesses a directional dependency. This implies that a mapping of damage induced by shear dominated fatigue has to be done in a local and multidirectional way. Both the amplitude and TOF recorded P-UPS results offer several indicators which can be used to assess shear dominated fatigue damage. It is found that the local fiber distortion is an important and straightforward indicator to monitor fatigue in shear loading for the carbon/ PPS laminate. The present results even suggest that the principal axes of 'orthotropy' for the fatigued sample are effectively rotated over $45^{\circ}$ due to the induced fiber distortion, which leads to a stiffening of the sample along the loading direction. The position $\theta_{Q L}$ of the inner contour, which is inversely proportional to the stiffness, has been extracted along $\varphi=0^{\circ}$ for all scan positions: $\left\{\theta_{Q L}^{R E F}, \theta_{Q L}^{A}, \theta_{Q L}^{B}\right.$,$\left.\theta_{O L}^{R E F}, \theta_{O L}^{A}, \theta_{O L}^{B}, \theta_{O L}^{C}, \theta_{O L}^{D}\right\}=\left\{18.1^{\circ}, 18.05^{\circ}, 17.6^{\circ}, 17.25^{\circ}, 14.2^{\circ}\right\}$. It is noted that equivalent results have been obtained for a carbon/PPS sample fatigued in a rail shear setup. For brevity, these results have been omitted.
Table 2 Ultrasonically and optically extracted local fiber orientation for the $\left[+45^{\circ},-45^{\circ}\right]_{4 \mathrm{~s}}$ carbon/ PPS fabric subjected to shear dominated fatigue loading

\begin{tabular}{llllll}
\hline Scan location & Virgin & A & B & C & D \\
\hline P-UPS & $-45^{\circ}$ & $-46^{\circ}\left(-1^{\circ}\right)$ & $-40(+5)$ & $-37^{\circ}\left(+8^{\circ}\right)$ & $-31^{\circ}\left(+14^{\circ}\right)$ \\
& $+45^{\circ}$ & $+44^{\circ}\left(-1^{\circ}\right)$ & $+42(-3)$ & $+37^{\circ}\left(-8^{\circ}\right)$ & $+34^{\circ}\left(-11^{\circ}\right)$ \\
Optical & $-45^{\circ}$ & $-45^{\circ}\left(0^{\circ}\right)$ & $-41^{\circ}\left(+4^{\circ}\right)$ & $-38^{\circ}\left(+7^{\circ}\right)$ & $-33^{\circ}\left(+12^{\circ}\right)$ \\
& $+45^{\circ}$ & $+44^{\circ}\left(-1^{\circ}\right)$ & $+42^{\circ}\left(-3^{\circ}\right)$ & $+38^{\circ}\left(-7^{\circ}\right)$ & $+36^{\circ}\left(-9^{\circ}\right)$ \\
\hline
\end{tabular}




\section{Tensile Fatigue Loading}

In analogy with previous paragraph, the influence of fatigue on the P-UPS image is investigated, but now for the carbon/ PPS sample with the $\left[0^{\circ}, 90^{\circ}\right]_{4 \mathrm{~s}}$ stacking sequence. First, the PUPS results for a virgin $[0,90]_{4 \mathrm{~s}}$ carbon/PPS sample are given as a reference (see Fig. 6). Obviously, these results correspond to the P-UPS results of the virgin $\left[+45^{\circ},-45^{\circ}\right]_{4 \mathrm{~s}}$ carbon/PPS sample, but rotated over an angle $\varphi=45^{\circ}$. In the $0^{\circ}$-direction, respectively $90^{\circ}$-direction, the inner contour is positioned at $\theta=13.88^{\circ}$, respectively $\theta=13.57^{\circ}$. This corresponds to a stiffness of $E_{x x}=54$ and $E_{y y}=57 \mathrm{GPa}$, which is in close agreement with literature $[43,45]$. The deviation between both stiffness values is a result of the difference in pre-stress between warp and weft yarns during the weaving.

Sample 1 was loaded at a frequency of $2 \mathrm{~Hz}$, with the longitudinal stress level varying between $0 \mathrm{MPa}$ and $700 \mathrm{MPa}$. The maximum applied stress corresponds to $90 \%$ of the ultimate tensile strength (UTS). The specimen withstood only 55 load cycles before failing and thus underwent lowcycle fatigue. The P-UPS results of the shear dominated specimen revealed the spatial distribution of fatigue damage (see Fig. 5), thus P-UPS scans are ideally performed at that spot where maximum material degradation occurred, obviously being the fractured region. However, this is not recommended here because geometrical discontinuities near the failed zone would obviously influence the P-UPS image, making a straightforward interpretation in terms of a reduction of material stiffness difficult. Therefore, the insonification spot is chosen at a distance of $d \approx 15 \mathrm{~mm}$ from the failed zone in order to avoid any 'edge' effects in the P-UPS image. Both the amplitude and TOF P-UPS scans for the 'fatigued' sample 1 are shown in Fig. 7(a-b). The amplitude map shows more detailed features in comparison with the TOF recording, therefore we focus the further discussion on the amplitude result. In addition, the TOF results of the fatigued samples do not reveal any significant change in its range, indicating the insensitivity of the TOF parameter for the material damage considered here.
Apart from a slight drop in transmitted amplitude, which can be attributed to the scattering of the ultrasonic waves on multiple matrix cracks, a slight directional stretching of the polar contours can be observed for the P-UPS image of sample 1. According to the physical background provided in section II, this positional shifting indicates a change in stiffness properties. After fatigue, the inner contour is positioned at $\theta=$ $14.38^{\circ}$ along $\varphi=0^{\circ}$, and $\theta=13.64^{\circ}$ along $\varphi=90^{\circ}$. Based on P-UPS simulations, we extracted a reduction of $E_{x x}$ of $3.7 \%$, while the stiffness modulus transverse to the loading direction remains more or less constant. Notwithstanding the very limited number of load cycles, a small stiffness reduction along the loading direction is obtained as could be expected for the carbon/PPS material [22, 38].

The P-UPS results of sample 2 (loading frequency $f=5 \mathrm{~Hz}$ and longitudinal stress [0-650 MPa]) show a larger drop in the global amplitude level, while the stretching of the inner contour becomes more outspoken (see Fig. 7(c-d)). The inner contour is now positioned at $\theta=15.23^{\circ}$ and $\theta=13.79^{\circ}$, along $0^{\circ}$-direction, respectively $90^{\circ}$-direction, affirming the directional nature of the tension-tension fatigue damage. A reduction of $10.5 \%$ is extracted for the stiffness along the loading direction. With respect to sample 1, the reduction in stiffness along the loading direction is larger, as could have been expected considering the higher number of load cycles. The extracted stiffness reduction along the loading direction is in full agreement with earlier observations reported in [22], where it was shown that the stress-strain response of the $\left[0^{\circ}, 90^{\circ}\right]_{4 \mathrm{~s}}$ laminate after high-cycle fatigue is nonlinear, showing a considerably lower stiffness for small strains, and a much higher stiffness for high strains. This is due to the geometrical stiffening of the laminate: due to the fatigue damage, the warp yarns are delaminated from the surrounding matrix and weft yarns, and can straighten up when tensile loading is applied. In case of ultrasonic inspection, the applied mechanical strains are negligible, so the stiffness is measured in the small-strain regime. In addition, the fatigue damage also influences the shear-related contour, causing the cross arms to split apart
Fig. 6 P-UPS result for virgin $\left[0^{\circ}, 90^{\circ}\right]_{4 \mathrm{~s}}$ carbon/PPS fabric: amplitude (a) and TOF (b) recording
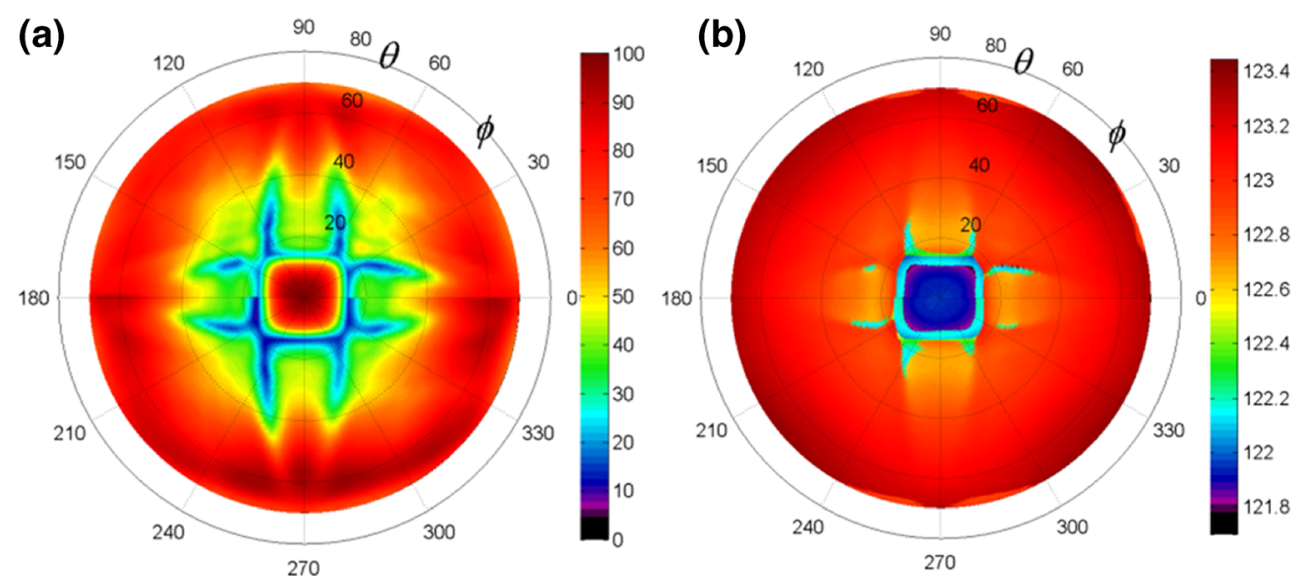
Fig. 7 Amplitude (left column) and TOF (right column) based PUPS results for tension-tension fatigued carbon/PPS fabric: sample $1[0 \mathrm{MPa}, 700 \mathrm{MPa}](\mathbf{a}-\mathbf{b})$ sample $2[0 \mathrm{MPa}, 650 \mathrm{MPa}]$ (c-d) and sample $3[0 \mathrm{MPa}, 625 \mathrm{MPa}]$ (e-f)
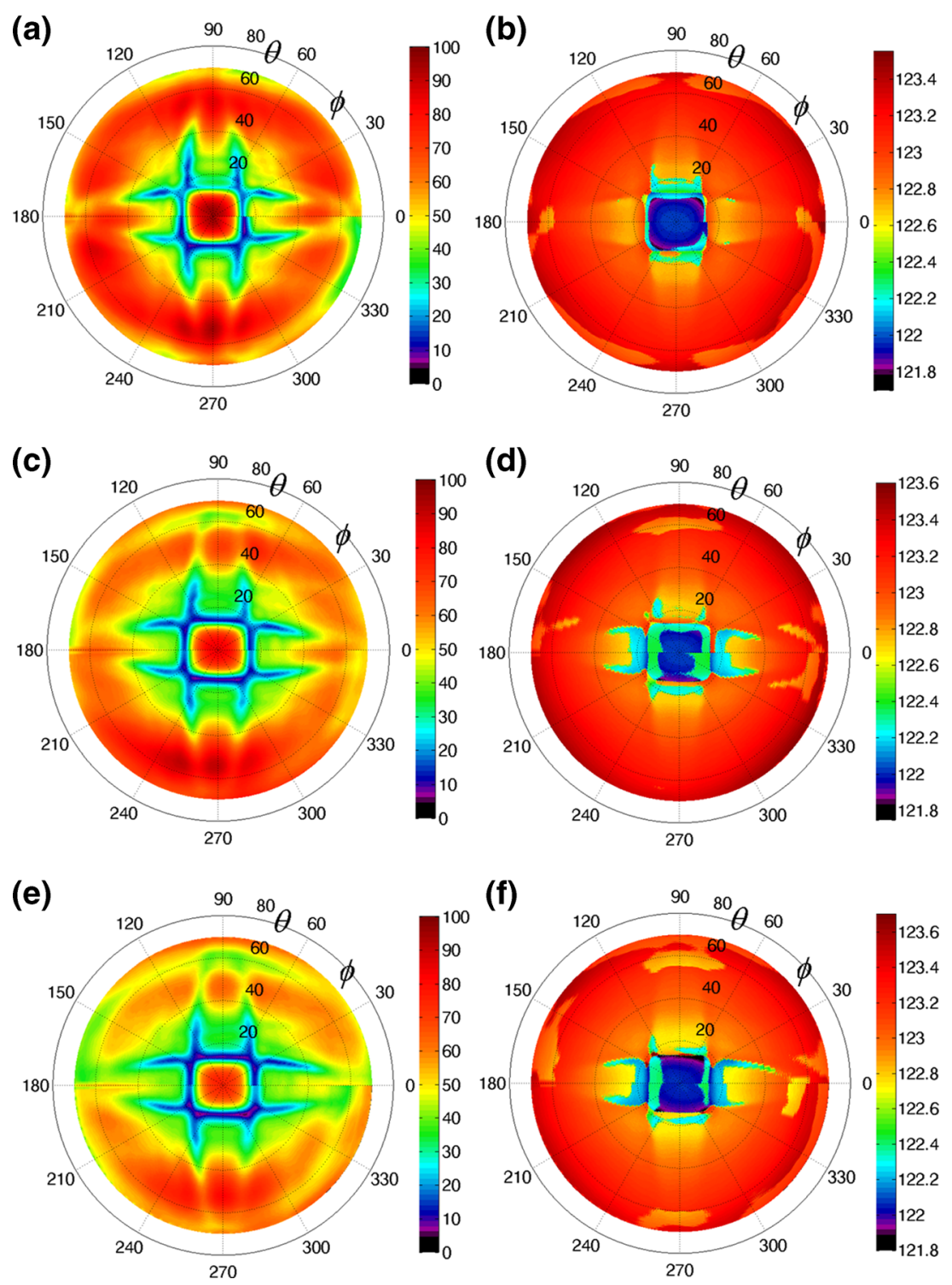

along the $90^{\circ}$-direction. As seen in the P-UPS simulation in which a reduced stiffness is considered (see Fig. 2(b)), this splitting is also observed. Considering that the particle displacement of the shear horizontally polarized bulk wave, guided by the structure along $\varphi=90^{\circ}$, is aligned with the tensile loading direction during fatigue, this splitting could have been expected.

Finally, sample 3 is fatigued at a loading frequency of $5 \mathrm{~Hz}$, with the tensile stress level varying between 0 and $625 \mathrm{MPa}$. After 314,111 load cycles, the specimen suddenly failed. The P-UPS results show very similar features as observed for sample 2. The inner contour is now positioned at $\theta=15.41^{\circ}$, respectively $\theta=13.56^{\circ}$, along $0^{\circ}$-direction, respectively $90^{\circ}$ direction. A stiffness reduction of $12.8 \%$ is extracted along the loading direction. As this sample was provided with an extensometer, we performed an additional analysis of the stress-strain data at small strains. A stiffness reduction of $\sim 11 \%$ has been extracted which is in good agreement with the P-UPS data.

The P-UPS results for the tensile fatigued carbon/PPS samples clearly reveal the directional nature of the stiffness reduction. The material degradation extracted from the P-UPS experiments correspond well to simulation, analysis of tensile data in the small strain area (sample 3 ) as well as to literature.

\section{Conclusions}

In this study, the experimental and numerical methodology of the non-destructive P-UPS technique has been applied to fatigued carbon fabric reinforced plastics. Several carbon/ PPS samples, subjected to either shear-dominated or tensiontension fatigue at various load levels, are investigated at 
multiple material spots with the P-UPS technique in order to assess fatigue damage.

For the carbon/PPS sample loaded in shear-dominated fatigue, the P-UPS results reveal the spatial and directional nature of the fatigue damage, and indicate that the shear properties of the fatigued carbon/PPS are subject to a reduction. In addition, the P-UPS results reveal large fiber distortions (up to $13^{\circ}$ ) at the place where necking was observed, suggesting that the principal axes of orthotropy could be rotated over $45^{\circ}$. On the basis of these results, one could actually question whether damage simulation models should be extended to account for fiber distortion and in extension to transformations in symmetry class.

The P-UPS results for the tension-tension fatigued carbon/ PPS samples show a reduction of stiffness up to $12.8 \%$ along the loading direction. Even the sample which was barely subjected to fatigue loading ( 55 cycles) did reveal a stiffness reduction of $3.7 \%$ along the loading direction. The P-UPS results for the tension-tension fatigued carbon/PPS samples do not reveal any significant change in stiffness transverse to the loading direction, confirming the directional nature of damage features associated to tension-tension fatigue.

Overall, good correspondence is obtained with simulations, conventional destructive tests, visual inspection as well as literature, indicating the excellent feasibility of the P-UPS technique to monitor and evaluate fatigue damage in fabric reinforced composites.

Acknowledgments Mathias Kersemans acknowledges funding of the FWO-Vlaanderen through grant G012010N. The authors are highly indebted to Ten Cate Advanced Composites for supplying the carbon/ PPS material.

\section{References}

1. Van den Abeele KEA et al (2000) Nonlinear elastic wave spectroscopy (NEWS) techniques to discern material damage, part I: nonlinear wave modulation spectroscopy (NWMS). Res Nondestruct Eval 12:17-30

2. Van den Abeele KEA et al (2000) Nonlinear elastic wave spectroscopy (NEWS) techniques to discern material damage, part II: singlemode nonlinear resonance acoustic spectroscopy. Res Nondestruct Eval 12:31-42

3. Armitage PR, Wright CD (2013) Design, development and testing of multi-functional non-linear ultrasonic instrumentation for the detection of defects and damage in CFRP materials and structures. Compos Sci Technol 87:149-156

4. Solodov IY et al (2002) CAN: an example of nonclassical acoustic nonlinearity in solids. Ultrasonics 40:621-625

5. Auld BA, Moulder JC (1999) Review of advances in quantitative eddy current nondestructive evaluation. J Nondestruct Eval 18:3-36

6. Liao TW, Ni JW (1996) An automated radiographic NDT system for weld inspection.1. Weld extraction. Ndt \& E International 29:157162
7. Liao TW, Li YM (1998) An automated radiographic NDT system for weld inspection: Part II - Flaw detection. Ndt \& E International 31: $183-192$

8. C. Ramadas, et al., "Interaction of the primary anti-symmetric Lamb mode $(\mathrm{A}(\mathrm{o}))$ with symmetric delaminations: numerical and experimental studies," Smart Materials \& Structures, vol. 18, Aug 2009.

9. Busse G et al (1992) Thermal wave imaging with phase sensitive modulated thermography. J Appl Phys 71:3962-3965

10. Manohar A, di Scalea FL (2013) Determination of defect depth and size using virtual heat sources in pulsed infrared thermography. Exp Mech 53:661-671

11. Kim JT, Stubbs N (2002) Improved damage identification method based on modal information. J Sound Vib 252:223-238

12. Kim JT et al (2003) Damage identification in beam-type structures: frequency-based method vs mode-shape-based method. Eng Struct 25:57-67

13. Carden EP, Fanning P (2004) Vibration based condition monitoring: a review. Struct Health Monit 3:355-377

14. Rippert L et al (2000) Optical and acoustic damage detection in laminated CFRP composite materials. Compos Sci Technol 60: 2713-2724

15. Y. Okabe, et al., "Delamination detection in composite laminates using dispersion change based on mode conversion of Lamb waves," Smart Materials \& Structures, vol. 19, Nov 2010.

16. Yashiro S et al (2007) An NDT technique for composite structures using visualized Lamb-wave propagation. Compos Sci Technol 67: 3202-3208

17. Dhital D, Lee JR (2012) A fully non-contact ultrasonic propagation imaging system for closed surface crack evaluation. Exp Mech 52: $1111-1122$

18. Kinra VK et al (2006) Ultrasonic ply-by-ply detection of matrix cracks in laminated composites. J Nondestruct Eval 25:39-51

19. Aymerich F, Meili S (2000) Ultrasonic evaluation of matrix damage in impacted composite laminates. Composites Part B-Engineering 31:1-6

20. Wooh SC, Wei C (1999) A high-fidelity ultrasonic pulse-echo scheme for detecting delaminations in composite laminates. Composites Part B-Engineering 30:433-441

21. Van Paepegem W et al (2010) Monitoring quasi-static and cyclic fatigue damage in fibre-reinforced plastics by Poisson's ratio evolution. Int J Fatigue 32:184-196

22. Daggumati S et al (2013) Fatigue and post-fatigue stress-strain analysis of a 5-harness satin weave carbon fibre reinforced composite. Compos Sci Technol 74:20-27

23. Taheri-Behrooz F et al (2008) Residual stiffness in cross-ply laminates subjected to cyclic loading. Compos Struct 85:205-212

24. Whitworth HA (1997) A stiffness degradation model for composite laminates under fatigue loading. Compos Struct 40:95-101

25. Hwang W, Han KS (1986) Cumulative damage models and multistress fatigue life prediction. J Compos Mater 20:125-153

26. Payan J, Hochard C (2002) Damage modelling of laminated carbon/ epoxy composites under static and fatigue loadings. Int J Fatigue 24: 299-306

27. Giancane $\mathrm{S}$ et al (2010) Characterization of fatigue damage in long fiber epoxy composite laminates. Int J Fatigue 32:46-53

28. Vandreumel WHM, Speijer JL (1981) Non-destructive composite laminate characterization by means of ultrasonic polar-scan. Mater Eval 39:922-925

29. Degrieck J et al (2003) Ultrasonic polar scans as a possible means of non-destructive testing and characterisation of composite plates. Insight 45:196-201

30. Declercq NF et al (2006) Simulations of harmonic and pulsed ultrasonic polar scans. Ndt \& E International 39:205-216

31. Degrieck J (1996) Some possibilities of nondestructive characterisation of composite plates by means of ultrasonic polar scans. 
Emerging technologies in nondestructive testing (ETNDT), Patras, pp 225-235

32. Kersemans M et al (2012) Quantitative measurement of the elastic properties of orthotropic composites by means of the ultrasonic polar scan method. JEC Composites 75:48-52

33. Kersemans $M$ et al (2014) Identification of the elastic properties of isotropic and orthotropic thin-plate materials with the pulsed ultrasonic polar scan. Exp Mech 54:1121-1132

34. M. Kersemans, et al., "Detection and localization of delaminations in thin carbon fiber reinforced composites with the ultrasonic polar scan, " Journal of Nondestructive Evaluation, vol. In Press DOI: 10.1007/ s10921-014-0249-5, 2014.

35. Kersemans M et al (2014) Nondestructive damage assessment in fiber reinforced composites with the pulsed ultrasonic polar scan. Polym Test 34:85-96

36. Kersemans M et al (2014) The pulsed ultrasonic backscatter polar scan and its applications for NDT and material characterization. Exp Mech 54:1059-1071

37. M. Kersemans, et al., "Ultrasonic Characterization of Subsurface 2D Corrugation," Journal of Nondestructive Evaluation, vol. In Press DOI: 10.1007/s10921-014-0239-7, 2014.

38. De Baere I et al (2011) On the tension-tension fatigue behaviour of a carbon reinforced thermoplastic part II: evaluation of a dumbbellshaped specimen. Polym Test 30:663-672
39. M. Kersemans, et al., "Extraction of bulk wave characteristics from a pulsed ultrasonic polar scan," Wave Motion, vol. In Press DOI: 10. 1016/j.wavemoti.2014.05.001, 2014.

40. J. L. Rose, Ultrasonic Waves in Solid Media: Cambridge University Press, 1999.

41. Declercq NF et al (2006) Ultrasonic polar scans: numerical simulation on generally anisotropic media. Ultrasonics 45:3239

42. M. Kersemans, et al., "The Quasi-Harmonic ultrasonic Polar Scan for material Characterization: Experiment and Numerical Modeling," presented at the Proceedings of the 12th International Conference for Non-Destructive Testing 2013 (ICNDT2013), Portoroz, Slovenia, 2013.

43. Daggumati $\mathrm{S}$ et al (2010) Local damage in a 5-harness satin weave composite under static tension: Part II - Meso-FE modelling. Compos Sci Technol 70:1934-1941

44. Sket F et al (2014) Automatic quantification of matrix cracking and fiber rotation by X-ray computed tomography in sheardeformed carbon fiber-reinforced laminates. Compos Sci Technol 90:129-138

45. Jacques S et al (2014) Application of periodic boundary conditions on multiple part finite element meshes for the meso-scale homogenization of textile fabric composites. Compos Sci Technol 92:41-54 